\title{
Global Citizenship Education und die Herausforderung epistemischer Dekolonialisierung
}

\begin{abstract}
Zusammenfassung
Zunehmend müssen sich pädagogische und erziehungswissenschaftliche Theorien und Konzepte mit dem Vorwurf auseinandersetzen, eurozentrische Perspektiven zu vertreten und dadurch koloniale Denkmuster zu reproduzieren. Deutlich zeigt sich dies in den Diskussionen über Global Citizenship Education, in denen daher auch Forderungen nach epistemischer Dekolonialisierung erhoben werden. Vor diesem Hintergrund versucht der vorliegende Beitrag exemplarisch an dem von der österreichischen UNESCO-Kommission herausgegebenen Konzept einer politischen Bildung für die Weltgesellschaft zu klären, wann und warum derartige Konzepte als eurozentrisch bezeichnet werden sollten, und wie ein solcher Eurozentrismus im Sinne epistemischer Dekolonialisierung überwunden werden kann. Dazu wird auf dekoloniale Theorien aus dem lateinamerikanischen Kontext zurückgegriffen. Dies führt zu der abschließenden These, dass diese Theorien als eine spezifische Form von Global Citizenship Education verstanden werden sollten, in der Sichtweisen des Globalen Südens artikuliert werden. Um die Herausforderung epistemischer Dekolonialisierung anzunehmen erscheint es daher empfehlenswert, auch im Globalen Norden stärker auf diese Theorien Bezug zu nehmen.
\end{abstract}

Schlüsselworte: Eurozentrismus, Kolonialismus, Kolonialität, Dekolonialität

\footnotetext{
Abstract

Increasingly, pedagogical and educational theories and concepts have to deal with the reproach of representing Eurocentric perspectives and thereby reproducing colonial thought patterns. This can also be clearly seen in the discussions on Global Citizenship Education, in which demands for epistemic decolonization are raised. Against this background, this contribution attempts to clarify, using the concept of Global Citizenship Education published by the Austrian UNESCO Commission as an example, when and why such concepts should be de-
}

scribed as Eurocentric, and how such Eurocentrism can be overcome in the sense of epistemic decolonisation. Decolonial theories from the Latin American context will be used for this purpose. This leads to the concluding thesis that these theories should be understood as a specific form of Global Citizenship Education, which articulates perspectives of the Global South. In order to meet the challenge of epistemic decolonization, it would therefore seem advisable to make greater reference to these theories in the Global North as well.

Keywords: eurocentrism, colonialism, coloniality, decoloniality

\section{Einleitung}

Pädagogische und erziehungswissenschaftliche Konzepte müssen sich mittlerweile zunehmend mit dem Vorwurf des Eurozentrismus auseinandersetzen. Besonders deutlich wird dies dort, wo durch Bezüge auf globale Phänomene bereits explizit oder implizit versucht wird, derartige Einschränkungen zu überwinden. Kritisiert werden so etwa Theorien und Materialien aus den Bereichen Globales Lernen und Bildung für Nachhaltige Entwicklung, denen aus dezidiert postkolonialer Perspektive unter anderem eine mangelnde oder unzureichende, letztlich (wenn überhaupt) eben eurozentrische Auseinandersetzung mit der Thematik des Kolonialismus attestiert wird (Danielzik, 2013; Castro Varela \& Heinemann, 2016). Als problematisch gilt dies vor allem dann, wenn auf diese Weise koloniale Denkmuster reproduziert werden, der Kolonialismus im Bereich des Epistemischen also gewissermaßen fortgesetzt wird. Folgt man dieser Sichtweise, so wird nachvollziehbar, warum etwa Vanessa de Oliveira Andreotti (2011) versucht, Konzepte von Global Citizenship Education zu dekolonialisieren, bzw. ein entsprechend dekoloniales Konzept zu entwickeln. Vor diesem Hintergrund überrascht es auch nicht, dass etwa in dem von der österreichischen UNESCO-Kommission herausgegebenen Konzept einer Global Citizenship Education (Wintersteiner et al., 2014) die Auseinandersetzung mit dem 
(europäischen) Kolonialismus als zentral und notwendig erachtet wird. Entsprechend wird auch auf die Gefahr verwiesen, dass Global Citizenship Education zum „Werkzeug eines neuen Imperialismus“ (Scherling \& Maurič, 2018, S. 11) werden könnte.

Nimmt man die Sorge hinsichtlich bestehender, verbreiteter und vermutlich sogar vorherrschender eurozentrischer Positionen und Perspektiven in Pädagogik und Erziehungswissenschaft ernst, und erkennt man in der epistemischen Dekolonialisierung eine drängende Aufgabe, so stellt sich mit Nachdruck die Frage, wie hier das ,koloniale Denken' (Forster, 2017) überwunden, wie also pädagogische Konzepte und erziehungswissenschaftliche Theorien dekolonialisiert werden können. Am Beispiel des von der österreichischen UNESCOKommission herausgegebenen und von Werner Wintersteiner, Heidi Grobbauer, Gertraud Diendorfer sowie Susanne Reitmair-Juaréz (2014) entwickelten Konzepts einer politischen Bildung für die Weltgesellschaft (bzw. Global Citizenship Education) soll in diesem Artikel daher untersucht werden, wann und warum eine derartige Pädagogik als eurozentrisch bezeichnet, und wie ein derartiger Eurozentrismus überwunden werden kann. Dazu werden einige zentrale Aspekte dieses Konzepts zunächst kurz vorgestellt.

\section{Global Citizenship Education}

Mit der Schrift Global Citizenship Education. Politische Bildung für die Weltgesellschaft hat die Österreichische UNESCO-Kommission eine Interpretation dieses Bildungskonzepts vorgelegt, die von Wintersteiner, Grobbauer, Diendorfer und Reitmair-Juárez im Zusammenhang eines gleichnamigen Universitätslehrgangs entwickelt wurde (Wintersteiner et al., 2014, S. 5). Für ein umfassendes Verständnis von Global Citizenship Education ist dies insofern interessant, als die UNESCO selbst ebenfalls im Jahr 2014 ein Dokument mit dem Titel Global Citizenship Education veröffentlichte. Dies deutet darauf hin, dass es zwar gewissermaßen allgemeine Grundlagen und Ansatzpunkte gibt, die weltweit einer entsprechenden pädagogischen Orientierung dienen können, es jedoch dennoch notwendig und sinnvoll ist, dieses Bildungskonzept vor dem Hintergrund des jeweiligen nationalen oder auch regionalen bzw. lokalen Kontextes zu interpretieren, zu konkretisieren und auch weiterzuentwickeln (UNESCO, 2018).

In dem von der Österreichischen UNESCO-Kommission herausgegebenen Global Citizenship Education-Konzept wird daher auch auf nationale bzw. lokale Referenzen verwiesen. Denn zwischen „Global Citizenship Education und anderen politischen Pädagogiken bestehen zwar gewisse Unterschiede, aber es gibt auch sehr viele Gemeinsamkeiten und Konvergenzen" (Wintersteiner et al., 2014, S. 28). Zu den politischen Pädagogiken, die zumindest teilweise ähnliche oder auch gleiche Ziele wie Global Citizenship Education verfolgen, werden hier die Politische Bildung, die Friedenspädagogik, das Globale Lernen, die Bildung für nachhaltige Entwicklung und das Interkulturelle Lernen bzw. die Interkulturelle Pädagogik gezählt (ebd., S. 28-35). Obwohl diese Pädagogiken natürlich nicht nur in Österreich oder im deutschsprachigen Raum zu finden sind und auch nicht nur dort entwickelt wurden, ist der Ver- weis doch insofern wichtig, als sie zumindest eben auch dort bekannt sind, und daher darüber ein Zugang zum Konzept Global Citizenship Education eröffnet werden kann. Entsprechend kann Global Citizenship Education als ein „sinnvoller Überbegriff“ (Grobbauer, 2014, S. 28) verstanden werden, der dazu dienen kann, die genannten und bekannten politischen Pädagogiken „miteinander zu verknüpfen“ (ebd.).

Ein zentraler Aspekt des von der Österreichischen UNESCO-Kommission vertretenen Global Citizenship Education-Konzepts ist die Unterscheidung zwischen individuell-humanitären und strukturell-politischen Ansätzen. Während individuell-humanitäre Konzepte auf die Bildung von Weltbürgerinnen und Weltbürgern im Rahmen der bestehenden globalen politischen Strukturen zielen, sollen strukturell-politische Konzepte dazu beitragen die Strukturen derart zu verändern, dass Formen einer Welt-Bürgerschaft (Global Citizenship) möglich werden. Betont wird dabei jedoch, dass diese Idee nicht auf die Errichtung eines Weltstaats zielt, sondern vielmehr einer Annäherung an das „Ideal einer friedlich organisierten, gerechten und demokratischen Weltgesellschaft “ (Wintersteiner et al., 2014, S. 13) dienen soll. Ein wichtiger Beitrag hierfür wäre etwa die weltweite Anerkennung der Menschenrechte. Neben dem UN-System zur „Regelung und Verrechtlichung der internationalen Beziehungen“ (ebd.) erscheint auch das Modell der Europäischen Union inspirierend, da hier nationalstaatliche Strukturen zumindest teilweise überwunden und neu integriert bzw. relationiert werden. Darüber hinaus würden bereits jetzt „Institutionen der international organisierten Zivilgesellschaft wie das World Social Forum oder das World Educational Forum“ (ebd.) dazu beitragen, ein „sehr konkretes politisches ,Weltbewusstsein“" (ebd.) auszubilden.

In Bezug auf die herausgestellte Differenzierung betonen Wintersteiner et al. (2014), dass sie selbst ein strukturell-politisches Konzept von Global Citizenship Education vertreten, in das sie aber auch Elemente des individuell-humanitären Ansatzes einfließen lassen wollen. Als besonders problematisch erachten sie es, wenn zwar einerseits die „Solidarität mit allen Menschen auf der Welt“ (ebd., S. 17) beschworen, die "Gleichheit aller Menschen“ (ebd., S. 18) betont sowie post- oder transnationale Identitäten und Gemeinschaften imaginiert, gerade dadurch aber andererseits strukturelle Ungleichheiten und Machtverhältnisse verdeckt werden: „Global Citizenship Education steht vor der Herausforderung, einerseits ein möglichst offenes und weltbürgerliches Konzept von citizenship zu entwerfen, andererseits aber nicht in unpolitische Denkmuster zu verfallen“ (ebd., S. 19).

Von großer Bedeutung ist für Wintersteiner et al. (2014) auch die Auseinandersetzung mit drei Grundfragen bzw. „häufig diskutierte[n] Probleme[n] jeder welt-bürgerlichen Bildung" (ebd., S. 21). Zu klären gelte es etwa (1.), wie das Verhältnis zwischen nationalen (bzw. regionalen oder anderen) und kosmopolitischen Identitäten konzipiert werde. Hier wird auf Martha Nussbaum verwiesen, die zwar einerseits dafür plädiert, an „traditionellen familiären, religiösen, ethnischen, kulturellen und staatlichen Bindungen“ (ebd.) festzuhalten, dabei jedoch andererseits auch anmahnt, dass dies heute nur noch möglich und sinnvoll sei, wenn dem Kosmopolitischen im Sinne einer umfassenden „Einheit der Menschheit“ (ebd.) dabei ein Vorrang eingeräumt werde. 
„Nussbaum plädiert also dafür, diese Einheit der Menschheit durch all ihre Vielfalt hindurch zu erkennen, ohne die Vielfalt aufzulösen, aber auch ohne sie enthusiastisch zu begrüßen" (ebd.). Auch hier betonen Wintersteiner et al., dass bei aller Betonung der Einheit der Menschheit die ,großen ungerechten Unterschiede in Bezug auf Wohlstand, Lebenschancen, Einflussmöglichkeiten" (ebd.) nicht übersehen werden dürfen. „Denn auch wenn wir den ,Anderen' als gleichwertig anerkennen, bleiben Unterschiede in den Interessen, Auffassungen und Ideologien, die nur politisch, also im demokratischen Streit, bearbeitet werden können" (ebd.).

Fraglich sei (2.) auch, wie eine Identität als Weltbürgerin bzw. Weltbürger ausgebildet werden soll, wenn es gar keinen Welt-Staat und entsprechend keine Welt-Staatsbürgerschaft gibt. Wintersteiner et al. (2014) halten es für wichtig, in Bezug auf diese Frage zwischen drei Formen von citizenship und entsprechend von drei Dimensionen von Global Citizenship Education zu unterscheiden. Denn citizenship könne sich auf einen rechtlichen Status, auf eine politische Praxis oder auf ein Gefühl der Zugehörigkeit beziehen. Während citizenship im Sinne einer Staatsbürgerschaft an einen bestimmten Nationalstaat gebunden ist, gebe es in der EU bereits transnationale Entwicklungen. „Alle EU-BürgerInnen haben bei Kommunalwahlen und bei den Wahlen zum Europäischen Parlament in dem Land, in dem sie leben, das aktive und passive Wahlrecht. Im Sinne von global citizenship kann für eine Ausweitung dieser Rechte plädiert werden, vielleicht in Richtung eines umfassenden eigenen EU-Bürgerrechts" (ebd., S. 22). Unabhängig von der jeweiligen Staatsbürgerschaft könnten Menschen auch Gefühle der (Nicht-)Zugehörigkeit zu einem - oder auch zu verschiedenen - Gemeinwesen entwickeln. Wichtig sei daher, dass alle MitbürgerInnen die Prinzipien von Demokratie und Menschenrechten verinnerlichen, um sich gerade auch an konflikthaften und spannungsreichen politischen Diskussionen beteiligen zu können: Denn citizenship sei auch eine Praxis: „Nur durch die eigene praktische Beteiligung können die Lernenden das Bewusstsein erlangen, dass sie eine Möglichkeit haben, den ,Lauf der Welt' zu beeinflussen“ (ebd., S. 23).

$\mathrm{Zu}$ diskutieren gelte es (3.) ebenso, in welcher Form Global Citizenship Education eine kritische Auseinandersetzung mit der eigenen Vergangenheit beinhalten kann bzw. soll. Dabei erachten es Wintersteiner et al. (2014) als besonders wichtig, gerade auch negative historische Ereignisse aufzugreifen und zu thematisieren, denn ,jedes Anknüpfen an die kosmopolitische und weltbürgerliche Tradition im europäischen Denken muss sich auch mit der dunklen Seite der europäischen Geschichte, mit Kolonialismus und Imperialismus, auseinandersetzen“" (ebd., S. 24). Bedeutsam sei für die Bildungsarbeit darüber hinaus auch „die Auseinandersetzung mit den beiden ,Zivilisationsbrüchen' des 20. Jahrhunderts, für die Ausschwitz und Hiroshima als Chiffren stehen" (ebd.).

Um die Auseinandersetzung mit dem Thema des Kolonialismus im Bereich von Global Citizenship Education zu begründen, reicht aber die Feststellung, dass es sich dabei um eine negative historische Tradition des Westens handelt, noch nicht aus. Darüber hinaus erscheint vielmehr zentral darauf hinzuweisen, dass dem Kolonialismus nicht nur eine historische, sondern auch eine paradigmatische Bedeutung zukommt. „Mit paradigmatischer Bedeutung ist gemeint, dass der Kolo- nialismus nicht nur ein politisches und ökonomisches Phänomen war, sondern auch eines, das die Ideologie und Ideenwelt der westlichen Zivilisation wesentlich bestimmt hat und bis heute bestimmt" (ebd., S. 25). In diesem Zusammenhang wird - unter Verweis auf den dekolonialen Denker Walter Mignolo - betont, dass der „uralte Rassismus“ (ebd.) im Zuge des europäischen Kolonialismus entwickelt wurde. „Auf diese Weise hat, wie Aimé Césaire feststellt, der Kolonialismus gerade die KolonisatorInnen, die sich als diejenigen verstanden, die den Barbaren die Zivilisation bringen, barbarisiert" (ebd.). Um die paradigmatische Bedeutung des Kolonialismus weiter zu erhellen, soll nachfolgend nun zunächst das Konzept der Kolonialität näher betrachtet werden. Das erscheint hinsichtlich der in diesem Artikel verfolgten Fragen vielversprechend, da mit dem Konzept der Kolonialität das Phänomen des Eurozentrismus aus der Perspektive des sogenannten Globalen Südens umfassend analysiert, und Möglichkeiten epistemischer bzw. epistemologischer Dekolonialisierung aufgezeigt werden können.

\section{Kolonialismus und Kolonialität}

Um die zuvor angesprochene paradigmatische Bedeutung des Kolonialismus für die Gegenwart genauer zu erschließen, ist es hilfreich, unter Rückgriff auf dekoloniale Theorien zwischen Kolonialismus und Kolonialität zu unterscheiden. Denn „Kolonialität ist [...], anders als Kolonialismus, ein fortwährendes Machtverhältnis, das mit der kolonialen Expansion Europas in die Amerikas entstand und als solches die Kehrseite sowie die notwendige Voraussetzung der westlichen Moderne darstellt" (Boatcă, 2016, S. 119). Geprägt wurde der Begriff der Kolonialität von dem peruanischen Soziologen Aníbal Quijano, der die Entwicklung der gegenwärtigen globalen Machtstrukturen mit dem modernen europäischen Kolonialismus in Verbindung bringt. „Die derzeitige Globalisierung ist zuallererst der Höhepunkt eines Prozesses, der mit der Konstitution Amerikas und des kolonialen/modernen eurozentrischen Kapitalismus als neuem Muster globaler Macht seinen Anfang nahm" (Quijano, 2016, S. 23).

Ein zentraler Aspekt dieses globalen Machtverhältnisses sei die „soziale Klassifizierung der Weltbevölkerung“ (ebd.), die im kolonialen amerikanischen Kontext mit der Unterscheidung zwischen Eroberern und Eroberten beginnt. Dabei sei für die Legitimierung des Herrschaftsverhältnisses zwischen Eroberern und Eroberten erstmals in der Geschichte die moderne Vorstellung von raza (bzw. ,Rasse') ${ }^{1}$ zur Anwendung gekommen. „Vielleicht bezog sie sich ursprünglich auf die phänotypischen Unterschiede zwischen Eroberern und Eroberten, aber wichtig dabei ist, dass sie sehr bald als Referenz für angeblich unterschiedliche biologische Strukturen zwischen diesen Gruppen konstruiert wurde“ (ebd., S. 26). Da sich die kolonialen Gesellschaften Amerikas in Bezug auf dieses neue Konzept von raza entwickelten, seien dort auch neue gesellschaftliche Identiäten, etwa die der Indios und Mestizen, entstanden. Die Kategorie der Hautfarbe sei laut Quijano vermutlich in den britisch-amerikanischen Kolonien erfunden worden, weshalb ab dann auch von (ausgebeuteten) Schwarzen die Rede war, und sich infolgedessen auch die Herrschenden als Weiße bezeichneten: „Im Verlauf der weltweiten Expansion kolonialer 
Herrschaft durch dieselbe herrschende raza - die Weißen (oder ab dem 18. Jahrhundert die Europäer), wurde das gleiche Kriterium gesellschaftlicher Klassifizierung der gesamten Weltbevölkerung aufgezwungen“" (ebd., S. 33).

Für Quijanos Analyse der Kolonialität der Macht ist entscheidend, dass während der Kolonialzeit eine Form der globalen Arbeitsteilung durchgesetzt wurde, die bestimmte Formen von Arbeit - sei es etwa Sklaverei, Leibeigenschaft oder Lohnarbeit - an spezifische razas koppelte. „In der Konsequenz konnte die Kontrolle einer spezifischen Arbeitsform zugleich die Kontrolle einer spezifischen Gruppe beherrschter Menschen sein. Eine neue Technologie der Herrschaft/Ausbeutung, in diesem Fall raza/Arbeit, vermittelte sich so, dass ihre Elemente naturgemäß zusammengehören schienen " (ebd.). Neu seien diese Kontrollformen insofern gewesen, als alle der Güterproduktion für den Weltmarkt dienten und über diesen miteinander koordiniert und verbunden waren. „So formierte sich eine neue, originale und singuläre Struktur von Produktionsverhältnissen in der historischen Erfahrung der Welt, der globale Kapitalismus“ (ebd., S. 31).

Europa gelang es nicht nur, bis ins 19. Jahrhundert hinein den Weltmarkt zu kontrollieren und zum Zentrum des globalen Kapitalismus zu werden, sondern bildete laut Quijano dadurch erst seine eigene, „neue geokulturelle Id-Entität“ (ebd., S. 35) - eben als modernes Europa - aus. Entstanden sei aber nicht nur eine neue kulturelle bzw. geokulturelle Vorstellung von Europa, sondern auch von den anderen Weltregionen. Denn durch die Kontrolle des Weltmarkts sei es möglich geworden, die koloniale (sowie postkoloniale und moderne) Herrschaft gewissermaßen auf die ganze Welt auszudehnen, und alle Regionen und Gesellschaften in dieses koloniale bzw. moderne kapitalistische Weltsystem einzubinden.

Durch die je nach Region unterschiedlichen Möglichkeiten der Kontrolle und Einflussnahme haben sich die Europäer mit den dortigen Kulturen laut Quijano auf drei unterschiedliche Weisen auseinandergesetzt bzw. versucht auf sie Einfluss zu nehmen: Während sich die Europäer (1.) die kulturellen Hervorbringungen der nicht-europäischen Völker aneigneten, die ihnen nützlich erschienen, versuchten sie (2.) die kulturellen Elemente zu unterdrücken, die sie für nicht brauchbar hielten. Darüber hinaus hätten sie (3.) versucht, Elemente ihrer eigenen Kultur dann unter der kolonisierten Bevölkerung zu verbreiten, wenn es ihnen vorteilhaft erschien (ebd., S. 42 f.). Diese führte dazu, dass ,schließlich alle kulturellen Erfahrungen, Geschichten, Ressourcen und Produkte in eine einzige globale kulturelle Ordnung eingebunden" wurden, die "sich um die europäische oder westliche Hegemonie“ (ebd., S. 42) drehte. Entsprechend entstanden auch ganz spezifische geokulturelle Vorstellungen, etwa von Afrika, Asien, Ozeanien oder dem Orient, die einerseits eurozentrische Perspektiven widerspiegeln, und andererseits weltweit Verbreitung fanden. Das Konzept der Kolonialität der Macht bezieht sich daher nicht nur auf die Bereiche der Politik und der Ökonomie, sondern auch auf die der Kultur, des Wissens und der Bildung. „Der gesamte [...] Prozess implizierte langfristig eine Kolonisierung der kognitiven Perspektiven, der Produktionsweisen und der Art und Weise, materiellen oder intersubjektiven Erfahrungen Sinn zu verleihen, der Vorstellungswelt (imaginario), des Universums intersubjektiver Beziehungen in der Welt, kurz der Kultur“ (ebd., S. 43).

Diese eben auch kulturelle Kolonisierung der Welt und der Weltanschauungen führte letztlich zur globalen Verbreitung eines spezifischen Verständnisses des Verhältnisses zwischen Europa bzw. dem Westen und dem Rest der Welt. Laut Quijano war es das Zusammenspiel von einem kolonialen Ethnozentrismus mit einer universellen rassialisierten Klassifizierung der Weltbevölkerung, das auch zu einem spezifischen und neuen Verständnis der Geschichte führte. Während Europa demnach Zentrum und Höhepunkt der Geschichte sei, befänden sich alle anderen Völker bzw. Kulturen - aufgrund ihres Wesens und ihrer Natur - noch in der Vergangenheit. „Übereinstimmend mit dieser Perspektive wurden Modernität und Rationalität ausschließlich als europäische Erfahrungen und Erzeugnisse imaginiert" (ebd. S. 45).

Es erscheint naheliegend, die Vorstellung, dass die Moderne eine europäische Erfindung ist, zu der der Rest der Welt nichts beigetragen hat, als zentrales Merkmal einer eurozentrischen Weltanschauung zu bezeichnen. Denn von einer eurozentrischen Vorstellung kann hier nicht nur gesprochen werden, weil Europa hier sozusagen als Zentrum der Weltgeschichte angesehen wird (auch Dussel, 2013), sondern auch, weil diese Vorstellung vor allem die Sichtweise jener widerspiegelt, die sich in Bezug auf dieses Konzept der Moderne selbst als modern - und in diesem Sinne als europäisch - bezeichnen. Da dieses Bild von Europa und dem Rest der Welt im Zuge kolonialer europäischer Erfahrungen entwickelt wurde, und sich aufgrund einer auf kolonialen Strukturen aufbauenden europäischen Vorherrschaft im Bereich von Kultur und Bildung weltweit verbreitet hat, erscheint es sinnvoll und notwendig, im Rahmen von Global Citizenship Education nicht nur das Thema des Kolonialismus, sondern vor allem auch das der Kolonialität aufzugreifen. Denn nur durch die Auseinandersetzung mit der bis heute wirkmächtigen Kolonialität der Macht wird es möglich, nicht nur über den Kolonialismus als historisches Phänomen zu informieren, sondern auch über seine paradigmatische Bedeutung für gegenwärtig vorherrschende eurozentrische Weltbilder und Denkformen aufzuklären. Global Citizenship Education könnte somit dazu beitragen, gerade auch uns selbst über die Kolonialität unseres Denkens aufzuklären. Da die Kolonialität und der damit verbundene Eurozentrismus im Bereich des Epistemischen ein globales und damit alle Weltbürgerinnen bzw. Weltbürger verbindendes Phänomen ist, sollte es möglich sein, durch diese Problemdiagnose ein kosmopolitisches Bewusstsein zu vermitteln, das auf gemeinsamen Interessen der Weltbürger gründet, ohne dabei globale Machtund Herrschaftsverhältnisse sowie deren Reproduktions- und Legitimierungslogik aus dem Blick zu verlieren.

\section{Moderne und Kolonialität}

Wenn Global Citizenship Education eurozentrische Einseitigkeiten in der politischen Bildung überwinden und koloniale Denkmuster - im Sinne einer epistemischen Dekolonialisierung - vermeiden und abbauen will, so nimmt der Rückgriff auf das Konzept der Kolonialität sicherlich eine zentrale Rolle ein. Dies stellt aber insofern eine große Herausforderung dar, 
als dieses Konzept einerseits sehr komplex ist, und sich andererseits den vorherrschenden Sichtweisen und Denkmustern, die im beschriebenen Sinne als eurozentrisch bezeichnet werden können, (noch) weitgehend entzieht. Daher überrascht es nicht, dass es beispielsweise im deutschsprachigen Raum mitunter (noch) große Probleme bereitet, ein klares Verständnis von Kolonialität und Dekolonialität zu gewinnen und zu vermitteln (Knobloch, 2019).

Die mit dem Begriff der Kolonialität verbundene hohe Komplexität lässt sich dadurch erklären, dass mit diesem letztlich nicht weniger als ein umfassendes Verständnis der Moderne vermittelt werden soll. Denn der Begriff der Kolonialität wird im dekolonialen Denken gewissermaßen als Synonym für den der Moderne verwendet. In diesem Sinne spricht Mignolo (2012) davon, dass Moderne und Kolonialität als zwei Seiten einer Medaille verstanden werden müssen. Die Differenzierung zwischen Moderne und Kolonialität ist dennoch wichtig, da die Kolonialität insofern von der Moderne zu unterscheiden ist, als sie, und zwar in zweifachem Sinne, die dunkle Seite der Moderne bezeichnet.

Als dunkel kann diese Seite der Moderne zunächst im Sinne einer moralischen Bewertung bezeichnet werden. Der Begriff der Kolonialität steht dann für negativ bewertete Ereignisse und Entwicklungen, die mit dem europäischen Kolonialismus und dem darauf aufbauenden modernen kapitalistischen Weltsystem in Zusammenhang stehen. In diesem Sinne wird der Begriff der Kolonialität verwendet, um auf eine Vielzahl an miteinander verwobenen Formen und Phänomenen politischer, ökonomischer, sozialer und kultureller Kontrolle, Unterdrückung oder Ausbeutung zu verweisen. Die andere Seite der Kolonialität, sozusagen die helle Seite der Moderne, wird dann entsprechend mit positiv bewerteten Entwicklungen und Errungenschaften assoziiert, etwa mit Aufklärung, Emanzipation, Demokratie und Menschenrechten. Laut Mignolo ist es für eurozentrische Sichtweisen typisch, streng zwischen der hellen und dunklen Seite der Moderne zu unterscheiden, um sich von dieser zu distanzieren, und sich mit jener zu identifizieren.

Von einer dunklen Seite der Moderne spricht Mignolo andererseits auch um darauf hinzuweisen, dass das bislang vorherrschende Konzept der Moderne nicht nur aus eurozentrischer Perspektive verfasst wurde, sondern dabei auch andere Sichtweisen auf die Moderne ausgeblendet wurden. In diesem Sinne liegen diese daher, wenn sie überhaupt schon artikuliert werden konnten, (noch) im Dunklen. „Die Moderne ist die Erzählung imperialer, sprachmächtiger Subjekte, die von ihrer eigenen Geschichte berichten und ihre Zugehörigkeit zu einer Region verschleiern" (ebd., S. 113). Die dekoloniale Theorie versucht daher die Sichtweise jener zur Sprache zu bringen, die im Sinne des vorherrschenden eurozentrischen Weltbildes (je nach Kontext) als nicht modern, also nicht zivilisiert, nicht aufgeklärt, nicht emanzipiert etc. verortet wurden und werden. Dabei spielt der Begriff der Kolonialität eine zentrale Rolle, da er eben auch erklären soll, was die Moderne für jene bedeutet, die an den Rändern der Moderne leben (müssen) und unter der kolonialen Matrix der Macht, also unter Formen moderner bzw. kolonialer Unterdrückung und Ausbeutung, leiden. Da die epistemische Dekolonialisierung in vielen sozialen und kulturellen Bereichen und wissenschaftlichen Disziplinen erst seit
Kurzem mehr Aufmerksamkeit erfährt, sind weitere wichtige Beiträge zum Verständnis der Kolonialität zu erwarten. Gerade deshalb erscheint auch die Auseinandersetzung mit Kolonialismus, Eurozentrismus und Kolonialität im Rahmen von Global Citizenship Education sinnvoll und notwendig.

\section{Grenzdenken und epistemische Dekolonialisierung}

Die bisherigen Überlegungen sprechen eindeutig für die Forderung von Wintersteiner et al. (2014), bei Global Citizenship Education nicht nur an die europäische Tradition des aufgeklärten kosmopolitischen Denkens anzuknüpfen, sondern dabei auch dunkle Seiten der europäischen Geschichte wie den Kolonialismus in kritischer Absicht zur Sprache zu bringen. Aus der Auseinandersetzung mit der Logik der Kolonialität (Mignolo, 2012; Knobloch, 2019) kann jedoch auch der Schluss gezogen werden, dass es nicht ausreicht, die negativen Aspekte des modernen europäischen Kolonialismus einfach ergänzend zur positiven Geschichte europäischer Emanzipation und Aufklärung zu thematisieren und zu kritisieren, wenn eurozentrische Sichtweisen überwunden werden sollen. Dies liegt daran, dass gerade die Kritik am Kolonialismus in der Entwicklung des Eurozentrismus eine entscheidende Rolle spielt.

Für den Eurozentrismus, wie er von Quijano (2016), Mignolo (2012) oder Dussel (2013) verstanden wird, ist die Kritik am (spanischen) Kolonialismus insofern konstitutiv, als sich die europäische Aufklärung nicht nur gegen die negativen Seiten der europäischen Kolonialherrschaft in Amerika wendet, sondern gerade dadurch auch selbst an Kontur gewinnt. Das lässt sich darauf zurückführen, dass die Moderne „ein europäisches Phänomen darstellt, das sich in dialektischer Beziehung zu einer nicht-europäischen Alterität als seinem letzten Gehalt konstituiert hat" (Dussel, zitiert nach Mignolo, 2012, S. 58 f.). Identitätsbildend wirkt die Abgrenzung von kolonialen Gewalttaten, Ausbeutungsformen und Unterdrückungsmustern für ein modernes europäisches Selbstbewusstsein insofern, als durch den kritischen Verweis auf die koloniale Barbarei im Umkehrschluss eine Vorstellung von wünschenswerter und fortschrittlicher europäischer bzw. westlicher Zivilisation konturiert wird (Wallerstein, 2007; Sarmiento, 2007). Damit liefert aber gerade die Kritik am barbarischen Kolonialismus gute Gründe, die globale Vorherrschaft des zivilisierten Westens zu legitimieren. In diesem Zusammenhang spricht Mignolo (2012) von einer Rhetorik der Moderne, die einer Logik der Kolonialität folgt: „Die Exteriorität, das ,Außen' der Moderne, das genau genommen von der Rhetorik der Moderne konstruiert wird [...], muss im Namen des Fortschritts und der Moderne erobert, kolonialisiert, beherrscht und bekehrt oder aber eliminiert werden" (ebd., S. 93). Aus dekolonialer Perspektive ist die „europäische Weltbürgerlichkeit“ daher nicht nur „ein begleitendes und ideologisierendes Element der kolonialen Unterwerfung des Globalen Südens“ (Wintersteiner, 2019, S. 21). Denn darüber hinaus muss der europäische Kolonialismus auch als Voraussetzung zur Entwicklung des europäischen Kosmopolitismus betrachtet werden. 
Für die Planung einer kritischen Auseinandersetzung mit Kolonialismus und Eurozentrismus im Rahmen von Global Citizenship Education ist sicherlich der Hinweis der dekolonialen Theorie zentral, dass es einen entscheidenden Unterschied macht, ob man sich von der hellen (bzw. westlichen) oder der dunklen (bzw. nicht westlichen) Seite der Moderne aus kritisch mit der Moderne und ihren Schattenseiten auseinandersetzt. Dabei ergänzen sich die beiden Perspektiven insofern, als sie eine radikale Kritik an der jeweils anderen Position ermöglichen. Dadurch zeigt sich aber auch, wie sowohl westliche als auch nicht-westliche kritische Positionen in die koloniale Matrix der Macht verstrickt sind.

Um zu verhindern, dass Global Citizenship Education koloniale Denkmuster reproduziert, indem entweder eurozentrische oder aber anti-aufklärerische und anti-westliche Positionen verklärt und absolutiert werden, erscheint es wichtig, sich am dekolonialen Grenzdenken zu orientieren, wie es etwa von Mignolo gefordert wird. Dieser verortet das Grenzdenken an „jener Schranke, die Moderne und Kolonialität voneinander trennt und miteinander verbindet" (Mignolo, 2012, S. 200).

Für die Ausrichtung von Global Citizenship Education bedeutet das natürlich vor allem, dass auf eine kritische Auseinandersetzung mit dem europäischen Kolonialismus nicht verzichtet werden darf. Ebenso wichtig wie der Rückgriff auf kosmopolitische, emanzipatorische, machtkritische - kurz: moderne - europäische Denktraditionen erscheint dabei aus dekolonialer Perspektive jedoch auch die Aufklärung darüber, wie dieses moderne kritische Denken bis heute in die koloniale Matrix der Macht verstrickt ist. Zur epistemischen Dekolonialisierung kann ein solches Grenzdenken insofern beitragen, als hier neben den bekannten und bisher vorherrschenden kritischen Theorien westlicher Provenienz nun auch kritische Denkformen aufgegriffen werden müssen, deren Ursprünge auf der dunklen Seite der Moderne zu suchen sind. Damit zeigt sich deutlich, dass kritisches und aufklärendes Denken kein Alleinstellungsmerkmal des Westens (mehr) ist.

\section{Fazit}

Zusammenfassend kann man festhalten, dass sich Konzepte und Programme der Global Citizenship Education dem Vorwurf des Eurozentrismus stellen und die Herausforderung epistemischer Dekolonialisierung annehmen sollten. Dies ist vor allem deshalb notwendig, da eurozentrische und koloniale Denkmuster nicht einfach überwunden werden können, schon gar nicht allein dadurch, dass man sich rhetorisch gegen Eurozentrismus und Kolonialismus positioniert. Um die epistemische Dekolonialisierung voranzutreiben, erscheint es vielmehr aussichtsreich, nicht nur den europäischen Kolonialismus, sondern vor allem auch Kolonialität und Dekolonialität zu zentralen Themen von Global Citizenship Education zu erheben. Dafür ist es jedoch notwendig, auch kritische Theorien aus dem Globalen Süden - wie beispielsweise dekoloniale Theorien aus dem lateinamerikanischen Kontext - aufzugreifen, und sowohl ergänzend als auch in kritischer Konfrontation mit westlichen Konzepten in die kosmopolitisch orientierte politische Bildungsarbeit zu integrieren. In diese Richtung weisen auch die aktuellen Überlegungen von Wintersteiner (2019), in de- nen eine "kosmopolitisch-dekoloniale Perspektive“ (ebd., S. 24) in der Politischen Bildung eingefordert wird. Global Citizenship Education beinhaltet demnach nicht nur die „kritische, dekoloniale Überprüfung westlicher Bildungskonzepte“ (ebd., S. 23), sondern auch die Auseinandersetzung mit „vielfältigen, allerdings keineswegs immer ,westlich-demokratischen' Formen des Widerstands politischer Bewegungen im Süden“ (ebd., S. 25; vgl. dazu auch Knobloch, 2019).

Vor dem Hintergrund der Gegenüberstellung von Konzepten politischer Bildung aus dem Globalen Süden und Norden stellt sich nun jedoch mit Nachdruck die Frage nach dem Stellenwert der dekolonialen Theorien für die aktuellen Diskussionen über Global Citizenship Education. Denn es erscheint nun nicht mehr zwingend, europäische bzw. westliche Traditionen des kosmopolitischen Denkens als Grundlage und Ausgangspunkt für eine global ausgerichtete politische Bildung zu nehmen, in der der Kolonialismus und die auf diesen bezogenen kritischen Theorien aus dem Globalen Süden dann auch eine (periphere) Rolle spielen bzw. spielen können. Vielmehr erscheint es nun ebenfalls möglich, dieses asymmetrische Verhältnis zwischen kritischen Theorien und Konzepten aus dem Globalen Norden und Süden in der politischen Bildung umzukehren. Dazu müsste man von kritischen Traditionen des kolonialen bzw. dekolonialen Denkens ausgehen, um dann von hier aus auch Konzepte kosmopolitischen Denkens aus dem Globalen Norden einzubeziehen. Dadurch könnte ein Verständnis von Global Citizenship Education entwickelt werden, das - im Sinne eines Grenzdenkens - zwischen kritischen Perspektiven aus dem Globalen Norden und Süden oszilliert, und dabei sowohl Gemeinsamkeiten wie auch Unterschiede reflektiert.

Diese Überlegungen führen zu dem Schluss, dass es sich bei den dekolonialen Theorien ebenfalls um Konzepte von Global Citizenship Education handelt, wenn auch sozusagen avant la lettre. Nicht übersehen werden sollte dabei jedoch, dass sich diese hinsichtlich ihrer epistemologischen Grundlagen mitunter deutlich von Konzepten unterscheiden, die als westlich oder eurozentrisch bezeichnet und mit dem Globalen Norden assoziiert werden können. Daher sollten nicht nur die Licht- und Schattenseiten der modernen europäischen, sondern ebenso der modernen/kolonialen nicht westlichen Traditionen des kosmopolitischen Denkens zum zentralen Thema von Global Citizenship Education gemacht werden. Wenn diese Sichtweise in den Diskussionen über Global Citizenship Education stärker berücksichtigt wird, wäre zumindest schon ein kleiner Beitrag zur epistemischen Dekolonialisierung politischer Bildung geleistet.

\section{Anmerkungen}

1 Ich verwende hier den Begriff raza in Anlehnung an die Überlegungen von Alke Jenss und Stefan Pimmer (vgl. Quijano, 2016, S. 23). 


\section{Literatur}

Andreotti, V. d. O. (2011). (Towards) decoloniality and diversality in global citizenship education. Globalisation, Societies and Education, 9(3-4), 381-397. https:// doi.org/10.1080/14767724.2011.605323

Boatcă, M. (2016). Postkolonialismus und Dekolonialität. In K. Fischer, G. Hauck \& M. Boatcă (Hrsg.), Handbuch Entwicklungsforschung (S. 113-123). Wiesbaden: Springer VS. https://doi.org/10.1007/978-3-658-04790-0_11

Castro Varela, M. d. M. \& Heinemann, A. M. B. (2016). Globale Bildungsbewegungen - Wissensproduktionen verändern. Zeitschrift für internationale Bildungsforschung und Entwicklungspädagogik, 39(2), 17-22.

Danielzik, Ch.-M. (2013). Überlegenheitsdenken fällt nicht vom Himmel. Postkoloniale Perspektiven auf Globales Lernen und Bildung für nachhaltige Entwicklung. Zeitschrift für internationale Bildungsforschung und Entwicklungspädagogik, 36(1), 26-33.

Dussel, E. (2013). Der Gegendiskurs der Moderne. Kölner Vorlesungen. Wien \& Berlin: Turia + Kant.

Forster, E. (2017). Ausblick auf das Ende des kolonialen Denkens. In S. Vock \& R. Wartmann (Hrsg.), Ver-antwortung im Anschluss an poststrukturalistische Einschnitte (S. 187-206). Paderborn: Schöningh.

Grobbauer, H. (2014). Global Citizenship Education - Politische Bildung für die Weltgesellschaft. Zeitschrift für internationale Bildungsforschung und Entwicklungspädagogik, 37(3), 28-33.

Knobloch, Ph. D. Th. (2019). Zur Übersetzung der Grammatik und Rhetorik der Dekolonialität. In N. Engel \& S. Köngeter (Hrsg.), Übersetzung. Über die Möglichkeit, Pädagogik anders zu denken (S. 115-135). Wiesbaden: Springer VS. https://doi. org/10.1007/978-3-658-20321-4_7

Mignolo, D. W. (2012). Epistemischer Ungehorsam. Rhetorik der Moderne, Logik der Kolonialität und Grammatik der Dekolonialität. Wien \& Berlin: Turia + Kant.
Quijano, A. (2016). Kolonialität der Macht, Eurozentrismus und Lateinamerika. Wien, Berlin: Turia + Kant.

Sarmiento, D. F. (2007). Barbarei und Zivilisation, Das Leben des Facundo Quiroga. Frankfurt am Main: Eichborn.

Scherling, J. \& Maurič, U. (2018). Drei Eckpunkte von Global Citizenship Education. In H. Grobbauer, W. Wintersteiner \& S. Reitmair-Juárez (Hrsg.), Global Citizenship Education in der Praxis. Erfahrungen, Erfolge, Beispiele österreichischer Schulen (S. 10-11). Wien: Österreichische UNESCO-Kommission.

UNESCO (2018). Global Citizenship Education: Taking it local. Paris: UNESCO. Wallerstein, I. (2007). Die Barbarei der anderen. Europäischer Universalismus. Berlin: Wagenbach.

Wintersteiner, W. (2019). Global Citizenship Education - eine pädagogische Antwort auf die ,große Regression"? Zeitschrift für internationale Bildungsforschung und Entwicklungspädagogik, 42(1), 21-25.

Wintersteiner, W., Grobbauer, H., Diendorfer, G. \& Reitmair-Juárez, S. (2014). Global Citizenship Education. Politische Bildung für die Weltgesellschaft. Wien: Österreichische UNESCO-Kommission.

\section{Dr. Phillip D. Th. Knobloch}

ist Akademischer Rat a. Z. am Institut für Allgemeine Erziehungswissenschaft und Berufspädagogik (IAEB) der Technischen Universität Dortmund. Arbeitsschwerpunkte: Allgemeine, Vergleichende, Internationale und Interkulturelle Pädagogik und Erziehungswissenschaft. Aktuelle Forschungsschwerpunkte: Konsumästhetische Bildung, dekoloniale Bildung.

\section{UNSERE BUCHEMPFEHLUNG}

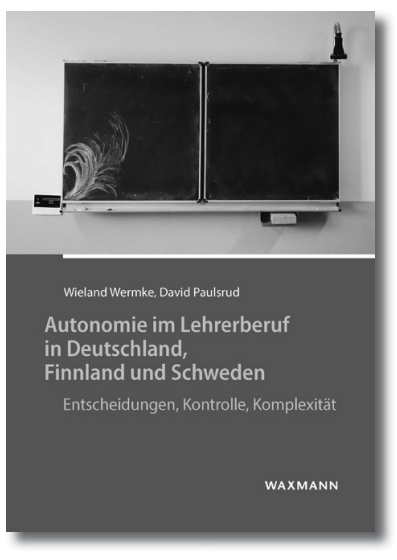

2019, 134 Seiten, br., 19,90€, ISBN 978-3-8309-4007-4

E-Book: $18,99 €$, ISBN 978-3-8309-9007-9
Wieland Wermke, David Paulsrud

\section{Autonomie im Lehrerberuf in Deutschland, Finnland und Schweden Entscheidungen, Kontrolle und Komplexität}

Basierend auf der Grundlage einer Umfrage unter ca. 7000 deutschen, 1500 finnischen und 700 schwedischen Lehrer*innen thematisieren die Autoren die Autonomie im Lehrerberuf. Bei der Diskussion über Autonomie geht es darum, Rahmenbedingungen zu schaffen, die Komplexität und Risiken reduzieren, um angstfreie und selbstbestimmte Arbeit in den Bereichen zu ermöglichen, in denen Lehrpersonen wichtige Entscheidungen treffen können und wollen. Beispiele aus den verschiedenen Bildungssystemen bereichern die Diskussion, die sowohl (angehende) Lehrer*innen betrifft als auch Entscheidungsträger*innen im Bildungsbereich, die Schulstrukturen gestalten. 